\title{
Molecular Dynamics Simulation of Self-Aggregation of Asphaltenes at an Oil/Water Interface: Formation and Destruction of the Asphaltene Protective Film
}

\author{
Juan Liu, ${ }^{\dagger, \dagger}$ Yapu Zhao, ${ }^{\S}$ and Sili Ren ${ }^{* \dagger}$ \\ ${ }^{\dagger}$ State Key Laboratory of Solid Lubrication, Lanzhou Institute of Chemical Physics, Chinese Academy of Sciences, Lanzhou 730000, \\ People's Republic of China \\ ${ }^{\ddagger}$ University of Chinese Academy of Sciences, Beijing 100039, People’s Republic of China \\ ${ }^{\S}$ The State Key Laboratory of Nonlinear Mechanics, Institute of Mechanics, Chinese Academy of Sciences, Beijing 100190, People’s \\ Republic of China
}

\section{Supporting Information}

\begin{abstract}
It is well known that asphaltene molecules play a significant role in stabilizing emulsions of water in crude oil or diluted bitumen solutions. Molecular dynamics simulations were employed to investigate the aggregation and orientation behaviors of asphaltene molecules in a vacuum and at various water surfaces. Two different continental model asphaltene molecules were employed in this work. It was found that the initially disordered asphaltenes quickly self-assembled into ordered nanoaggregates consisting of several molecules, in which the aromatic rings in asphaltenes were reoriented to form a face-to-face stacked structure. More importantly, statistical analysis indicates that most of the stacked polycyclic aromatic planes of asphaltene nanoaggregates tend to be perpendicular to the water surface. If the asphaltene molecules are considered as "stakes", then the asphaltene nanoaggregate can be regarded as a "fence". All the fence-like nanoaggregates were twined and knitted together, which pinned them perpendicularly on the water surface to form a steady protective film wrapping the water droplets. The mechanism of stabilization of the water/oil emulsions is thereby well understood. Demulsification processes using a chemical demulsifier were also studied. It was observed that the asphaltene protective film was destroyed by a demulsifier of ethyl cellulose molecules, leading to exposure of the water droplet. The results obtained in this work will be of significance in guiding the development of demulsification technology.
\end{abstract}

\section{INTRODUCTION}

As the heaviest fraction of crude oil or bitumen, asphaltenes are complex mixtures of molecules composed of aliphatic chains, polyaromatic condensed rings, organometallic complexes, and heteroatoms of nitrogen, oxygen, and sulfur. ${ }^{1}$ They are defined by their insolubility in low-boiling paraffinic solvents such as $n$ pentane and $n$-heptane and their solubility in toluene and benzene. $^{2}$ Asphaltenes are regarded as the most enigmatic component in petroleum because of their negative influence on stabilizing the water-in-crude oil emulsion, ${ }^{3-7}$ affecting the wettability of reservoir rock during oil recovery, ${ }^{8}$ plugging the reservoir pores and pipes in the transportation, ${ }^{9}$ corroding the metal surface of distillation equipment, ${ }^{10}$ and poisoning the catalysts ${ }^{11}$ during refinement processes. All these challenges are closely related to their self-aggregation.

In recent years, great efforts have been made to study the aggregation of asphaltenes and their effect on stabilizing oil/ water emulsions. Various analytical instruments were employed to study the asphaltene molecules' self-aggregation behaviors. For example, a photometric dispersion analyzer was used to investigate asphaltene aggregation online. ${ }^{12} \mathrm{~A}$ micropipet technique was widely used to investigate the formation and destruction of asphaltene molecular films at water/oil interfaces $^{13-16}$ as well as in demulsification processes. ${ }^{17,18}$ Moreover, after asphaltene films were transferred onto glass or silicon surfaces, the film morphology was examined by atomic force microscopy (AFM) in order to study how the demulsifiers destroy the asphaltene film. ${ }^{19-23}$ Previous studies greatly deepened our understanding of the emulsion stabilization and demulsification mechanism. However, experimental research has inevitable shortcomings in studying the aggregation of asphaltenes at nanoscale or molecular levels. ${ }^{24}$ Therefore, computer simulation techniques such as molecular mechanics (MM), molecular dynamics (MD), Monte Carlo (MC), Brownian dynamics (BD), and dissipative particle dynamics (DPD) simulations are useful complements to the experimental research. These simulations could well describe the dynamics of the evolution of molecular behaviors at water/oil interfaces and thereby reveal the nature of asphaltene aggregation. With the help of computer simulations, numerous studies on the selfaggregation of asphaltenes have been carried out at the molecular level in the past decade.

Rogel investigated the asphaltenes' aggregation in mixed solvents $^{25}$ and found that more-stable dimers of asphaltene aggregates were obtained as the ratio of $n$-heptane to toluene increased. They ascribed the stabilization energy of asphaltene aggregates mainly to the van der Waals interactions between molecules. ${ }^{26}$ Asphaltene aggregation in a vacuum at different

Received: September 3, 2014

Revised: January 19, 2015

Published: January 19, 2015 
temperatures was studied by Pacheco-Sánchez et al. ${ }^{27}$ It was suggested that different aggregate structures corresponded to different types of interaction. In another study by the same group, asphaltene aggregates in face-to-face stacking and $\pi$ offset or T-shaped stacking geometries were observed through a geometry optimization process in the molecular simulation. ${ }^{28} \mathrm{~A}$ further study on heat-induced relaxation of asphaltene aggregates was done by Takanohashi and co-workers. ${ }^{29}$ They found that the intermolecular hydrogen bonds between asphaltenes dissociated at $523 \mathrm{~K}$, but the aromatic-aromatic stacking configuration was still considerably stable. Sedghi and Goual et al. suggested that the interaction between stacked polycyclic aromatic hydrocarbons (PAHs) is the driving force for aggregation. The interaction strength is dependent on not only the number of aromatic rings but also the presence of heteroatoms in the aromatic core that can reduce the electrostatic repulsion. ${ }^{30}$ Ungerer et al. conducted the MD simulations of model asphaltene molecules in both toluene and $n$-heptane. Their studies indicated that asphaltenes underwent stronger stacking in $n$-heptane than in toluene. The aggregation process of asphaltene is apparently irreversible, which is attributed to the dispersion energy between the PAHs of asphaltenes. $^{31}$

The orientation of asphaltene aggregates in single-solvent or binary solvents system was also investigated by molecular simulations. Greenfield and Zhang ${ }^{32}$ found that the molecular orientation of neighboring molecules was closely related to the molecular structure and the environmental temperature. It was observed that asphaltene molecules with long aliphatic branches prefer to exhibit parallel arrangement at the high temperature of $443.15 \mathrm{~K}$, while they prefer to pack perpendicularly at the low temperature of $298.15 \mathrm{~K}$ and have an intersection angle about $40^{\circ}$ between molecules at the intermediate temperature of $358.15 \mathrm{~K}$. Highly aromatic asphaltene molecules prefer to stay almost parallel to each other at low temperatures and almost perpendicular at high temperatures. Bhattacharjee et al. studied the asphaltene-like structures in aqueous/organic solvent systems. ${ }^{33}$ The simulations showed that the model molecules tend to form aggregates in pure solvent, and specific fractions with ionic terminal groups on the aliphatic chains could dramatically enhance the interfacial activity of the entire asphaltene population. Such findings indicated that the interfacial activity of the asphaltene molecules was probably related to their terminal group characteristics rather than the polyaromatic rings. Aggregation and partitioning of the model asphaltenes at toluene/water interfaces were further studied by Bhattacharjee et al. ${ }^{34,35}$ It was observed that the polyaromatic rings of the asphaltene molecules prefer to position themselves perpendicularly to the toluene/water interface. Boek et al. ${ }^{36}$ investigated the interfacial phenomena of oil/asphaltene/water systems. Their simulations showed that the PAHs plane is attracted by the interfacial water molecules to form stable parallel structures. The angles between the aromatic planes of two types of asphaltene and the oil/water interface at various times were also calculated. Their statistics indicated that the nanoaggregate of asphaltenes could oscillate and adopt a vertical structure with respect to the oil/water interface.

Destroying the stability of crude oil emulsions is an important step in the petroleum industry. A few simulations were also performed on the demulsification process of the model crude oil emulsion. For example, demulsification of heavy crude oil emulsions in the presence of a functionalized copolymer was investigated by Alvarez et al. by using the framework of the DPD simulation. ${ }^{37}$ It was found that the copolymer with short-chains was more effective to accelerate the water coalescence than that of with long chains. In this work, an all-atom model was employed by using MD simulation to study the aggregation of asphaltene molecules at the water/ oil interface. The molecular orientation of asphaltenes and how the asphaltene protective film formed at oil/water interface were investigated. Additionally, demulsification by chemical demulsifiers was also studied to illustrate the processes of how the protective film composed of asphaltene nanoaggregates was destroyed.

\section{MOLECULAR DYNAMICS SIMULATION}

Molecular dynamics simulations on aggregation behaviors of asphaltene molecules in vacuum or at various water surfaces were carried out to understand the water/oil emulsion system. The emulsion system consists of three parts: the water droplet, the asphaltene layer, and the continuous phase (vacuum or toluene). By changing the type of asphaltenes and continuous phase, we were able to investigate the aggregation and orientation of asphaltenes at the water surface.

The three-dimensional (3D) structures of molecules of water, toluene, asphaltenes, and ethyl cellulose (EC) were created and optimized by using the Accelrys Material Studio software package 4.4 (MS). The coordinates of molecular structures were then exported and submitted to the Packmol program ${ }^{38}$ to generate the predefined molecular configurations for the $\mathrm{MD}$ simulations. By geometrical optimization with the program, molecules were constrained within a defined area at specified positions to avoid the atoms overlapping or being too close to one another. Various initial predefined geometrical structures were created with the program, including spherical, cubic, or random. The cutoff distance was set to be $2.0 \AA$ in all the initial molecular configurations. The export files from the Packmol program were then submitted back to MS to obtain the proper molecular characters and combine the different shapes of molecular configurations to form even more complex simulation systems, such as the toluene/asphaltene/water system.

Despite the dispute over the structure of asphaltenes, it is generally accepted that asphaltene molecules can be classified into two architectures. The continental model is shaped like a hand, in which the aromatic rings are considered the palm and the alkyl side chains are the fingers. The other widely accepted model is the archipelago architecture, where each asphaltene molecule is composed of several fused aromatic rings linked by bridge chains. Recently, a quantitative molecular representation (QMR) technique ${ }^{39,40}$ based on experimental data was employed to obtain the structure of asphaltene molecules used in the simulations. In this work, two typical continental asphaltene molecular models were used in the simulations (Figure 1). ${ }^{41}$ The composition and structure of coal asphaltene and petroleum asphaltene molecules were inspired by the Indonesian bituminous coal sample and the UG8 Kuwait crude oil sample, ${ }^{42}$ respectively. The coal asphaltene has a pyridine ring, four condensed aromatic rings, and

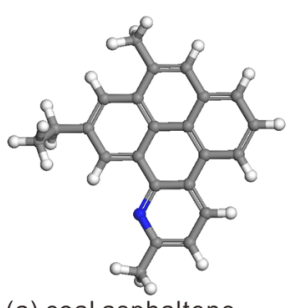

(a) coal asphaltene $\left(\mathrm{C}_{23} \mathrm{H}_{19} \mathrm{~N}\right)$

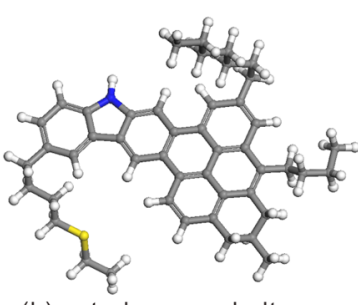

(b) petroleum asphaltene $\left(\mathrm{C}_{47} \mathrm{H}_{55} \mathrm{NS}\right)$
Figure 1. Three-dimensional chemical structures of the asphaltene molecules used in the simulations: (a) coal asphaltene and (b) petroleum asphaltene. 


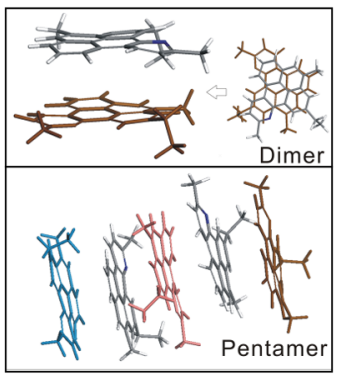

(a1)

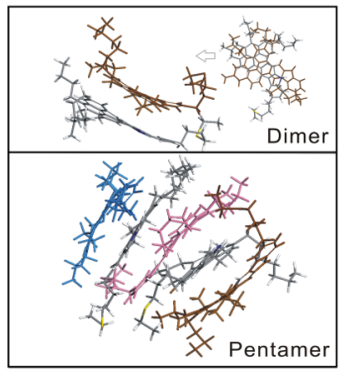

(b1)

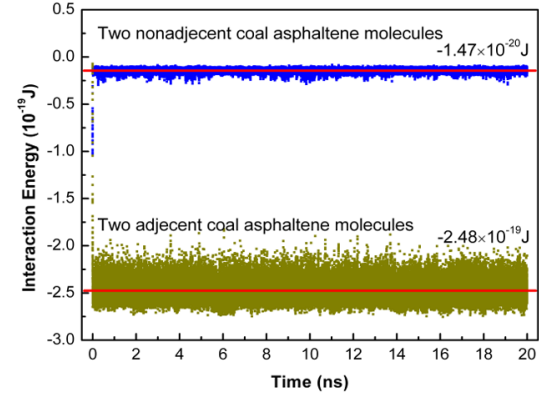

(a2)

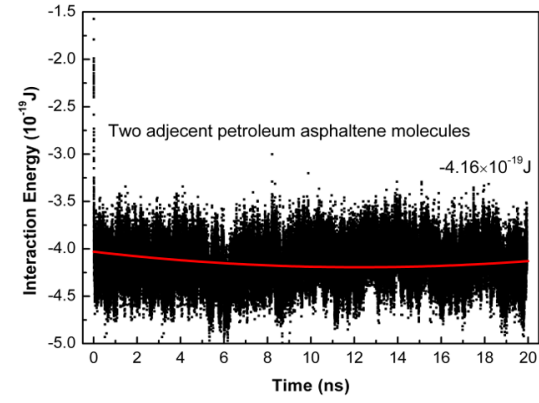

(b2)

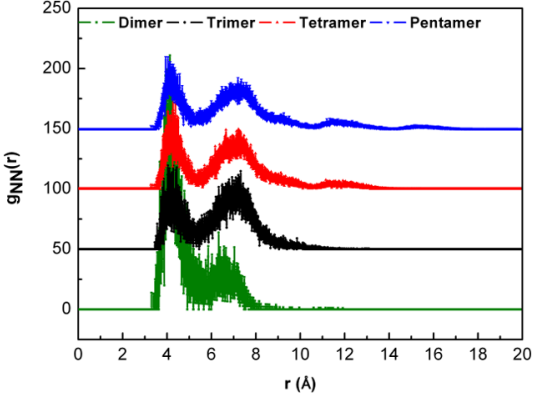

(a3)

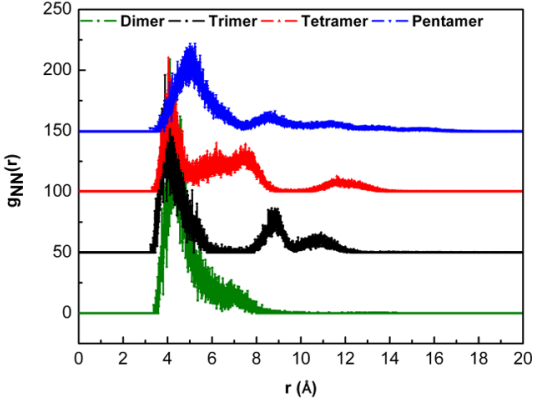

(b3)

Figure 2. Aggregates of asphaltenes in vacuum (a1, b1), interactions between two coal (a2) or petroleum (b2) asphaltene molecules, and the radial distribution function, $g(r)$, for aggregates of coal (a3) or petroleum (b3) asphaltenes at $300 \mathrm{~K}$.

three short alkyl chains. Meanwhile, the petroleum asphaltene has a single polyaromatic nucleus including a saturated cycle, a pyrrole ring, and six aromatic rings. Four alkyl chains are linked to the polyaromatic core. Similar asphaltene models have been used in the early MD simulations. ${ }^{29,34}$ The water molecules were modeled by the extended simple point charge potential (SPC/E) ${ }^{43}$ Prior to simulation, the water droplet was first relaxed for $10 \mathrm{~ns}$ at $300 \mathrm{~K}$. In the simulations, the oil phase was represented by toluene. Consistent valence force field $(\mathrm{CVFF})^{44}$ was used to describe the asphaltene and other molecules. In a previous study, CVFF correctly described the formation of dimers for a series of aromatic compounds from benzene to coronene. ${ }^{26}$ The charges of the atoms were assigned according to the CVFF database and remained constant during the calculations.

The MD simulations were performed by using the large-scale atomic/molecular massively parallel simulator (LAMMPS), ${ }^{45}$ which is an open source program for massively parallel simulations. All the simulations were carried out in the NVT ensemble using a NoséHoover thermostat at ambient temperature of $300 \mathrm{~K}^{46}$ The nonbonded interactions between the sites were described by the Lennard-Jones potential, with the cutoff of $1.1 \mathrm{~nm}$. The long-range electrostatic interactions were handled using the particle-particleparticle-mesh (PPPM or P3M) method with a cutoff distance of 1.1 $\mathrm{nm}$ and an accuracy of 1 part in $1 \times 10^{4}$ for all simulations. A $1.0 \mathrm{fs}$ time step was used, and the output trajectories were recorded every 0.1 ps. In all the simulations, one minimization algorithm for highly overlapped systems was employed to minimize the local potential energy of the simulation system during all the simulation processes by iteratively adjusting the atom coordinates. Iterations are terminated when one of the stopping criteria (such as energy, force, the maximum iterations, and the maximum number of force/energy evaluations) is satisfied. The simulation box sizes and calculation steps were chosen according to the given system. During the model construction and simulations, 3D periodic boundary conditions were applied. In the simulations, the system energy and the force between molecular groups were monitored to indicate the equilibrium of the systems (details are given in the Supporting Information). All the molecular configurations and snapshots were visualized and acquired with visual molecular dynamics (VMD). ${ }^{47}$

In order to validate the molecular model and force field, a series of additional simulations were conducted to obtain the physical properties of the water, toluene, and asphaltenes. The obtained physical properties, including the density and diffusion coefficients, were compared with the reported calculations and experimental data. It was found that the bulk densities of the water and toluene obtained in the simulations were 984.1 and $867.4 \mathrm{~g} / \mathrm{L}$, respectively, which are in good agreement with the experimental data (the densities are $997.07^{48}$ and $\left.862.3 \mathrm{~g} / \mathrm{L}^{49}\right)$. In addition, the diffusion coefficients of various molecules in the simulations were calculated by using the Einstein equation. ${ }^{50}$ The bulk self-diffusion coefficient of water is $2.42 \times 10^{-9}$ $\mathrm{m}^{2} / \mathrm{s}$, which is close to the reported experimental value of $(2.27-2.57)$ $\times 10^{-9} \mathrm{~m}^{2} / \mathrm{s} .{ }^{51,52}$ Meanwhile, the bulk self-diffusion coefficient of toluene is $2.49 \times 10^{-9} \mathrm{~m}^{2} / \mathrm{s}$, which is good agreement with the value of $2.29 \times 10^{-9} \mathrm{~m}^{2} / \mathrm{s}$ in the previous simulation. ${ }^{35}$ The diffusion coefficients of the coal and petroleum asphaltenes in toluene are found to be $5.14 \times 10^{-11}$ and $3.87 \times 10^{-11} \mathrm{~m}^{2} / \mathrm{s}$, respectively, which agree with the reported experimental results of $(2-7.5) \times 10^{-11} \mathrm{~m}^{2} /$ s. ${ }^{33}$ On the basis of these results, it is believed that the molecular modes and force field used in the simulations could well reflect the physical properties of various molecules (refer to the Supporting Information, Figures S1 and S2).

\section{RESULTS AND DISCUSSION}

3.1. Aggregation of Asphaltenes. The asphaltene aggregates generally have a variety of molecular stacking modes of dimers, trimers, tetramers, pentamers, etc. The aggregation behaviors of a few asphaltene molecules in vacuum/toluene were first studied. Two model molecules of the coal and petroleum asphaltenes (Figure 1) with $\mathrm{N}$ atom or $S$ atom labeled were used in the simulations. Asphaltenes molecules were randomly set into a cubic simulation box edges of $5 \mathrm{~nm}$. Aggregation of the coal and petroleum asphaltene molecules in vacuum/toluene was conducted at $300 \mathrm{~K}$ for 20 ns. Taking the asphaltene dimer and pentamer aggregates as examples, two and five asphaltene molecules were randomly distributed in vacuum/toluene initially. When the systems reached their equilibrium, it was found that the asphaltene molecules aggregated spontaneously and formed dimer or pentamer aggregates with the aromatic ring sheets face-to-face 
stacked together. Figure 2al depicts the dimer and pentamer aggregates of the coal asphaltenes in vacuum, in which the aromatic rings are stacked together. As for aggregates of the petroleum asphlatenes, the aromatic rings experienced a deformation to form the stacked configurations because of the steric effect of aliphatic groups (Figure 2b1). The energy of the random asphaltene molecules was reduced by forming the aggregates. The stabilization energy for the aggregates was calculated from the difference between the total energy of the aggregates and the sum energy of the monomers (Figure S3). ${ }^{26}$ The average stabilization energies for various aggregates in vacuum and toluene are listed in Table 1 . It was found that

Table 1. Stabilization Energy for Various Asphaltene Nanoaggregates in Vacuum and Toluene

\begin{tabular}{|c|c|c|c|c|c|}
\hline & & \multicolumn{4}{|c|}{ stabilization energy $\left(10^{-19} \mathrm{~J}\right)$} \\
\hline & & dimer & timer & tetramer & pentamer \\
\hline \multirow[t]{2}{*}{$\begin{array}{l}\text { coal asphaltene, } \\
\mathrm{C}_{23} \mathrm{H}_{19} \mathrm{~N}\end{array}$} & $\begin{array}{l}\text { in } \\
\text { vacuum }\end{array}$ & -2.48 & -5.15 & -7.93 & -10.08 \\
\hline & $\begin{array}{l}\text { in } \\
\text { toluene }\end{array}$ & -2.39 & -4.92 & -7.38 & -9.89 \\
\hline \multirow{2}{*}{$\begin{array}{l}\text { petroleum } \\
\text { asphaltene, } \\
\mathrm{C}_{47} \mathrm{H}_{55} \mathrm{NS}\end{array}$} & $\begin{array}{l}\text { in } \\
\text { vacuum }\end{array}$ & -4.16 & -8.38 & -12.71 & -17.19 \\
\hline & in ${ }_{\text {toluene }}$ & -4.02 & -8.05 & -12.54 & -17.06 \\
\hline
\end{tabular}

there is no obvious distinction between the stabilization energies of asphaltenes in the two situations. It can be seen that the stabilization energy increased with increasing numbers of asphaltene molecules, indicating that the asphaltene molecules achieve their energy-minimum configuration by aggregating each other.

The interaction energy between two asphaltene molecules in vacuum was also calculated. The interaction energies for two coal or petroleum asphaltene molecules are shown in Figure $2 \mathrm{a} 2, \mathrm{~b} 2$. It is found that the energy plots are convergent and stable with increasing simulation times. The average interaction energies for two coal or petroleum asphaltene molecules are $-2.48 \times 10^{-19}$ or $-4.16 \times 10^{-19} \mathrm{~J}$, respectively. The interaction energy between two asphaltene molecules is equal to the corresponding stabilization energy of the asphaltene dimer aggregate. The energy minimization is mainly due to the interactions between adjacent molecules. In contrast, the interactions between two nonadjacent coal asphaltene molecules (e.g., the blue and the pink asphaltene molecule in Figure 2a1) are extremely small compared with those of adjacent molecules (about $1.47 \times 10^{-20} \mathrm{~J}$, the blue plot in Figure 2a2).

To get a better understanding of the aggregation of asphaltenes, the aggregate structure was analyzed using the radial distribution function (RDF), $g(r)$, which gives the probability of the occurrence of a particle "a" at a distance $r$ from particle "b", compared to the average number density. The RDF was calculated through the following equation,

$$
g(r)=\frac{1}{\rho 4 \pi^{2} \delta r} \frac{\sum_{t=1}^{T} \sum_{j=1}^{N} \Delta N(r \rightarrow r+\delta r)}{N T}
$$

where $\rho$ is the density of the system, $N$ is the number of atoms, $T$ is the total time of the simulation, $\delta r$ is the interval distance as set initially, and $\Delta N$ is the atom number between $r$ and $r+\delta r$. Using the $\mathrm{N}$ atom on the aromatic rings as a reference point, a cutoff radius of $30 \AA$, and an interval distance of $0.02 \AA$, the RDF plots were calculated through the trajectory file data exported by LAMMPS. The RDF plots for the coal and petroleum asphaltene aggregates are shown in Figure 2. It is believed that the peaks on the plots associated with specific neighbor molecules could well reflect the microscale configuration of an aggregate. For example, two peaks appearing on the $g(r)$ plot for the aggregate of two coal asphaltene molecules indicate that probably there are two different configurations for the aggregate (the green plot in Figure 2a3). The two peaks are located at about 4.1 and $6.3 \AA$, respectively. However, the petroleum asphaltene molecules with aliphatic groups tend to form only one configuration of the dimer aggregate because of the steric effect of the aliphatic groups. As a result, only one peak appears on the $g(r)$ plot (the green plot in Figure 2b3), and the distance between two adjacent PAHs planes is about $4.3 \AA$. The $g(r)$ plots for aggregates of the trimer and tetramer are also shown in Figure 2a3,b3. Some small peaks also appeared besides the main peaks, which might be assigned to the nonadjacent molecules. However, the distance between two PAHs is slightly larger than the reported value of $3.8 \AA$, $^{31,54}$ which is attributed to the fact that the aromatic rings of two asphaltene molecules are not completely overlapped and the distance between two $\mathrm{N}$ atoms of asphaltenes would be larger than the real distance of two PAHs planes.

It is well known that natural surfactants such as asphaltenes and resins in petroleum tend to aggregate at the oil/water interface, ${ }^{5}$ which has attracted considerable attention in recent years. Disputes are focused on the orientations of the plane of the aromatic rings, i.e., whether they are parallel, perpendicular, or at a certain angle with the liquid/liquid interfaces. ${ }^{35,36,55,56}$ Therefore, simulations were performed with a number of coal or petroleum asphaltenes at the water surface.

Because the critical micelle concentration (CMC) of various asphaltenes in toluene was in a large range of $0.1-3.24 \mathrm{~g} / \mathrm{L}, 57,58$ it is difficult to select a proper asphaltene concentration in the simulation system. In order to study the aggregate behavior of asphaltenes at the water surface, sufficient asphaltene molecules $(\gg \mathrm{CMC}$ ) were used in the systems. In addition, it was reported that the diffusion coefficient of Athabasca asphaltenes in toluene was in the range of $2-7.5 \times 10^{-11} \mathrm{~m}^{2} / \mathrm{s}^{53}$ Although the diffusion time of asphaltene molecules from the oil phase to

Table 2. Simulation Conditions Sets for Asphaltenes' Aggregation at Water Surface

\begin{tabular}{|c|c|c|c|c|c|}
\hline simulation & continuous phase $/ N / \rho(\mathrm{g} / \mathrm{L})$ & asphaltene & $N_{\text {asphaltene }} / \rho(\mathrm{g} / \mathrm{L})$ & $N_{\text {water }} / R_{\text {water }}(\mathrm{nm}) / \rho(\mathrm{g} / \mathrm{L})$ & time (ns) \\
\hline 1 & vacuum & coal & 100 & $8960 / 4 / 1000$ & 2 \\
\hline 2 & vacuum & coal & 200 & $3780 / 3 / 1000$ & 2 \\
\hline 3 & vacuum & coal & 400 & $3780 / 3 / 1000$ & 6 \\
\hline 4 & vacuum & petroleum & 200 & $3780 / 3 / 1000$ & 6 \\
\hline 5 & toluene $/ 4500 / 870$ & coal & $400 / 205.3$ & $3780 / 3 / 1000$ & 5 \\
\hline 6 & toluene and $\mathrm{EC} / 4500$ and $50 / 870$ and 123.6 & coal & $400 / 205.3$ & $3780 / 3 / 1000$ & 2 \\
\hline
\end{tabular}



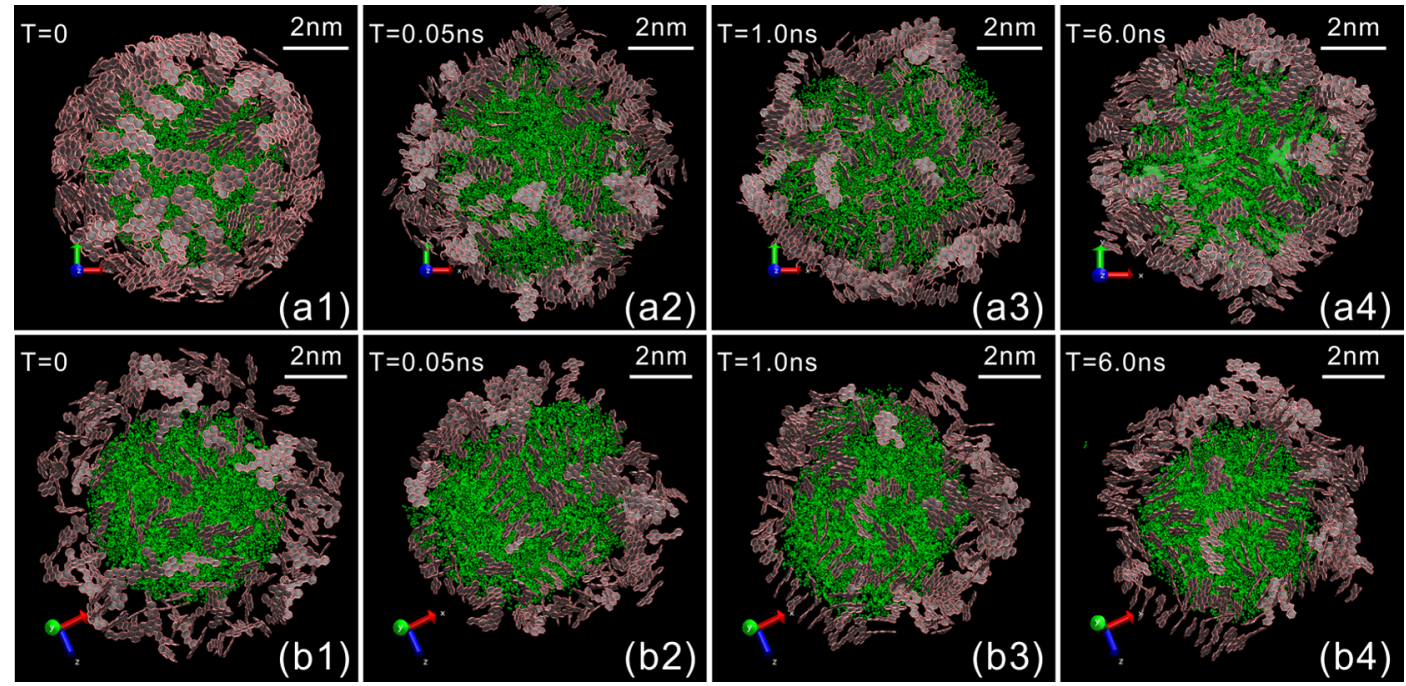

Figure 3. Sample snapshots of the coal $(\mathrm{a} 1-\mathrm{a} 4)$ and petroleum $(\mathrm{b} 1-\mathrm{b} 4)$ asphaltene molecular behavior on the water surface at various time intervals. Water and asphaltene molecules are represented in dark green and pink, respectively.
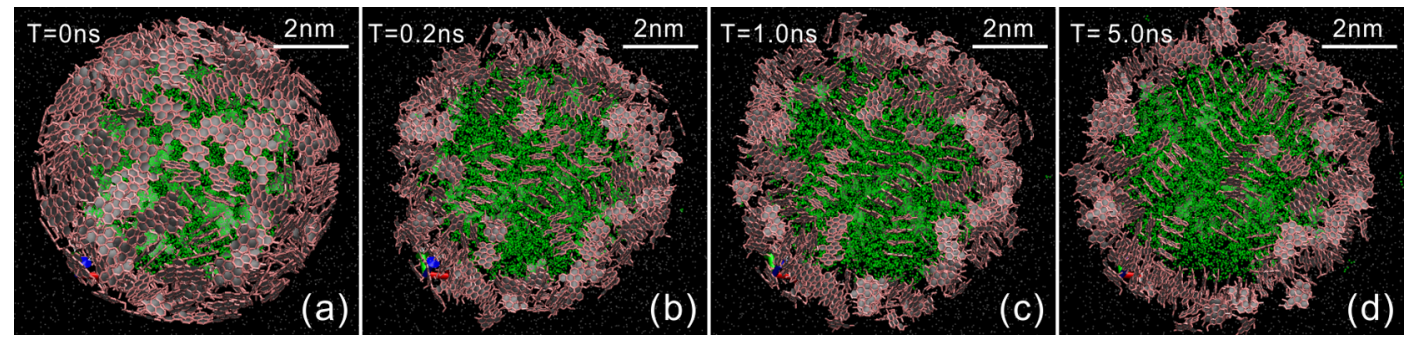

Figure 4. Sample snapshots of the coal asphaltene molecular behavior at the toluene/water interface at various time intervals. Water, toluene, and asphaltene molecules are represented in green, white, and pink, respectively.

the oil/water interface is short in practice, it would be a very long time in the simulation. To save calculation time, the asphaltene molecules were randomly placed in a shell with a specific radius from the water surface. The density of the oil phase was designed to be close to the actual condition. In the simulation system, a water droplet wrapped with asphaltene molecules was placed at the center of the continuous phase in a simulation box with edges of $10 \mathrm{~nm}$. The MD simulation was conducted for a sufficient time to reach their equilibrium state. Details of the simulation system setup, including the molecular number, density, and simulation time, are listed in Table 2.

The aggregations of the coal and petroleum asphaltenes were initially performed at the vacuum/water interface, and the results are shown in Figure 3 (corresponding to simulations 3 and 4 in Table 2). In order to observe the simulation system and molecular behavior more clearly, only part of the carbon skeleton in the molecules is shown, and other atoms such as hydrogen atoms are hidden. It is found that the initially disordered coal or petroleum asphaltene molecules quickly selfassembled into an ordered structure at the water surface when the system reached its equilibrium, indicated as a function of the system energy with simulation time (Figures S4 and S5). A graphite-like hierarchical structural aggregate consisting of a few asphaltene molecules was formed. More importantly, it is interesting that most of the stacked aromatic ring sheets preferred to be perpendicular to the water surface. Moreover, the stacked structure remained essentially stable for the rest of the simulation time (up to $6 \mathrm{~ns}$ ). It is observed that only a few water or asphaltene molecules flew away from the water surface in the simulation process when the system was placed in vacuum. It should be pointed out that the aggregation and orientation of the asphaltenes at the water surface did not result from steric effects because of a high molecular density. For example, the asphaltene molecules still showed the same aggregation behavior for the situation with only 100 molecules on a larger water droplet surface (simulation 1 in Table 2).

The aggregation and orientation of the coal asphaltenes at a toluene/water interface were also investigated. As shown in Figure $4 \mathrm{a}$, a water droplet $(1000 \mathrm{~g} / \mathrm{L})$ with a radius of $3 \mathrm{~nm}$ wrapped by 400 coal asphaltene molecules $(205.3 \mathrm{~g} / \mathrm{L})$ was immersed in toluene $(870 \mathrm{~g} / \mathrm{L})$. The water/asphaltene/toluene system was then placed in a cubic simulation box with edges of $10 \mathrm{~nm}$. The simulation was conducted for sufficient simulation time at $300 \mathrm{~K}$, indicated as a function of the system energy with the simulation time (Figure S6). The aggregation of asphaltenes at the toluene/water interface is shown in Figure 4. It is observed that the PAHs of coal asphaltenes are aggregated in parallel, and most of the PAHs plane tends to be perpendicular to the toluene/water interface after a simulation time of $0.2 \mathrm{~ns}$, which is analogous to observations with the asphaltenes at the vacuum/water interface (Figure 3). A further increase in simulation time did not cause further obvious changes in the parallel structure and the perpendicular orientation of the aggregates at the toluene/water interface (up to $5 \mathrm{~ns}$ ). This finding reflects that, once the asphaltene nanoaggegates were formed and reoriented themselves to be perpendicular with the water surface, the configuration remained stable and did not change for the rest of the 
simulation time. These results are in good agreement with previous simulations on asphaltenes aggregates at the flat toluene/water interface reported by Bhattacharjee et al. ${ }^{33-35}$ Mikami et al. ${ }^{36}$ found that the angle between the aromatic plane and the oil/water interface was closely related to the number of asphaltene molecules. Taking the island-type asphaltene molecules as an example, the asphaltene molecules could oscillate and adopt a vertical structure with respect to the oil/ water interface when the number of asphaltene molecules increased.

3.2. Orientation of PAHs with Respect to the Water Surface. To further investigate the preferred distribution and orientation of asphaltene molecules at the water surface, a quantitative analysis on the orientation of the asphaltenes was conducted by calculating the angle between the aromatic planes and the water surface. The inset of Figure 5 shows the

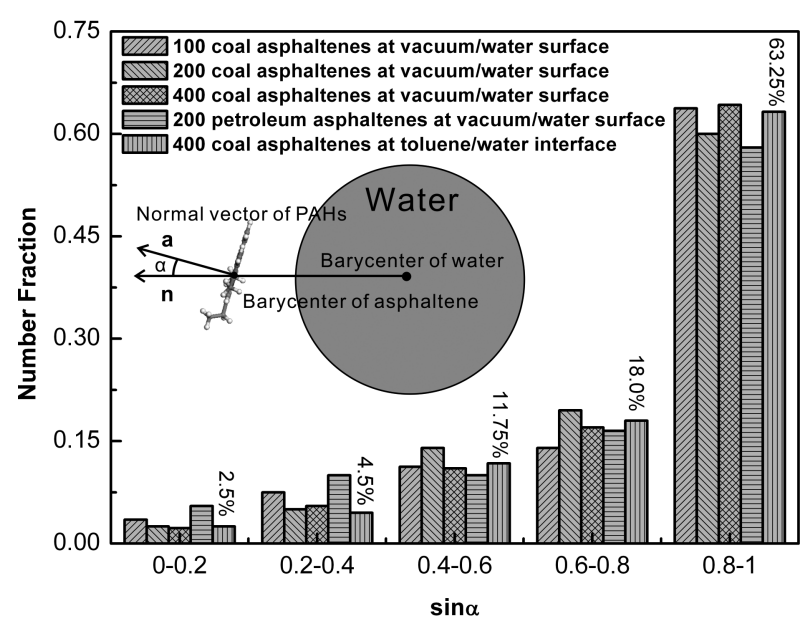

Figure 5. Histogram distribution of asphaltenes' orientation on various water surfaces. Inset: schematic depiction of the calculation of angle $\alpha$ between vectors $\mathbf{a}$ and $\mathbf{n}$, which are the normal vector of the asphaltene PAHs plane and the barycenter vector from water droplet to asphltene molecule, respectively.

methodology of calculation of angle $\alpha$ formed by vector $\mathbf{n}$, which is from the barycenter of the water droplet to the asphaltene molecule, and vector a, which is normal to the asphaltene PAHs plane. The angle $\alpha$ is equal to the inclination angle of PAHs with respect to the matching tangent of the water surface. To obtain the angle of inclination, the values of $\sin (\alpha)$ of every asphaltene molecule were calculated by employing the simulation trajectories. The calculation was performed for the last picosecond of each simulation. When the value of $\sin (\alpha)$ is close to 1 , the PAHs of asphaltenes will be perpendicular to the water surface. While $\sin (\alpha)$ is close to 0 , the aromatic planes will be parallel to the water surface. The distribution of the $\sin (\alpha)$ data for PAHs at various water surfaces is summarized in Figure 5. It can be seen that most of the asphaltene molecules orient themselves with the PAH planes nearly perpendicular to the water surface. Take the orientation of 400 coal asphaltenes at water/toluene interface as an example: about $63.25 \%$ of the asphaltene molecules are nearly perpendicular to the oil/water interface $(0.8 \leq \sin (\alpha) \leq$ $1.0)$, which is the predominant configuration of the asphaltene nanoaggregates at the water surface.

To have a better understanding on the asphaltenes' aggregation at various water surfaces, the RDF of the nanoaggregate configuration was also calculated and is shown in Figure 6. The RDF plots of the coal asphaltenes at vacuum/ water and toluene/water interfaces are shown in Figure 6a. It can be seen that the two plots (green and black) have similar tendencies, with the main peak located at about 4.1 A for both plots. However, on closer examination, a difference is found between the two plots. Compared to the situation of asphaltenes at the vacuum/water surface (the green plot), the $\mathrm{RDF}$ for asphaltenes at the toluene/water interface has some small peaks (the black plot). This might be attributed to the fact that toluene is a good solvent for asphaltenes. Although the asphaltene protective film could be still formed on the water surface which was not dissolved by the toluene, ${ }^{59,60}$ the fine internal configuration of the asphaltene may have been possibly influenced. The RDF for petroleum asphaltenes at the vacuum/ water surface is shown in Figure $6 \mathrm{~b}$ (the blue plot). It is found that the main peak is located at $4.3 \AA$, which is similar to that of the dimer aggregate shown in Figure 2b3. In contrast, the small peaks are not obvious, indicating that the petroleum asphaltene molecules could form well-stacked nanoaggregates at the vacuum/water interface.

3.3. From Nanoaggregates to Protective Film at the Oil/Water Interface. Formation of a stable asphaltene protective film at the oil/water interface has been observed by using the micropipet technique. ${ }^{15,61,62}$ The MD simulations clearly showed that the asphaltene molecules could well selfassemble to form ordered graphite-like aggregates in vacuum or at the vacuum/liquid and liquid/liquid interface (Figures 2-4).
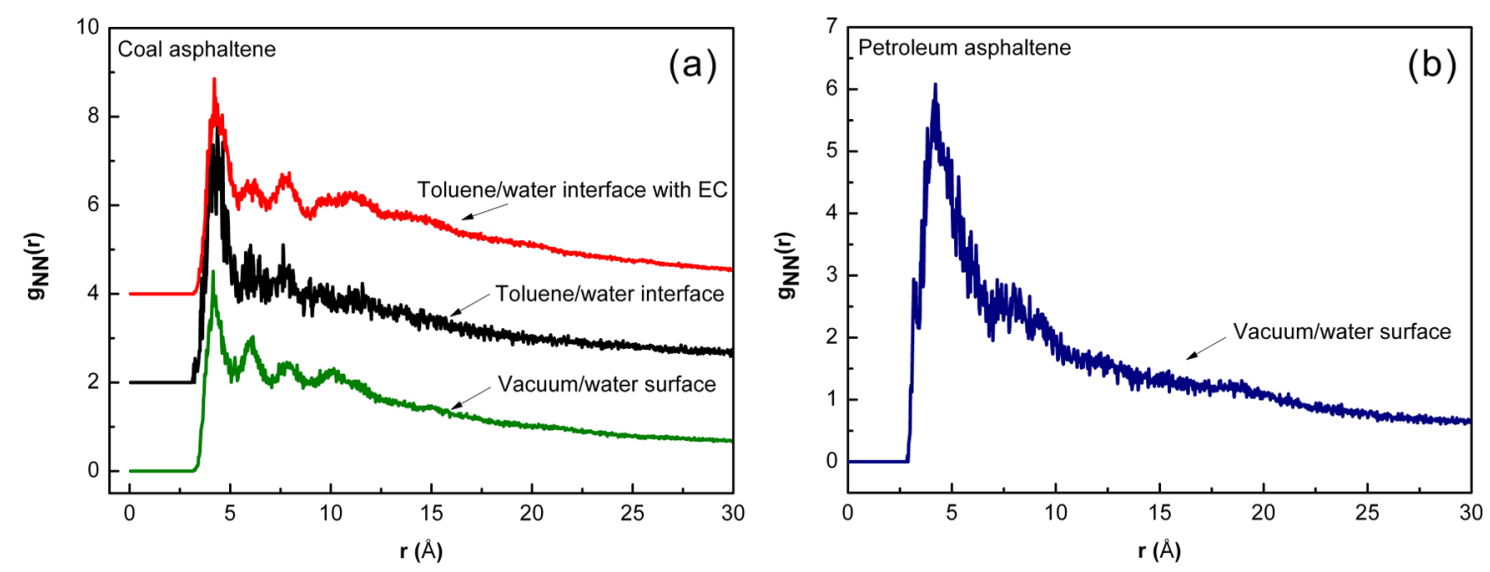

Figure 6. Radial distribution function, $g(r)$, for coal (a) or petroleum (b) asphaltene aggregates at various water surfaces. 

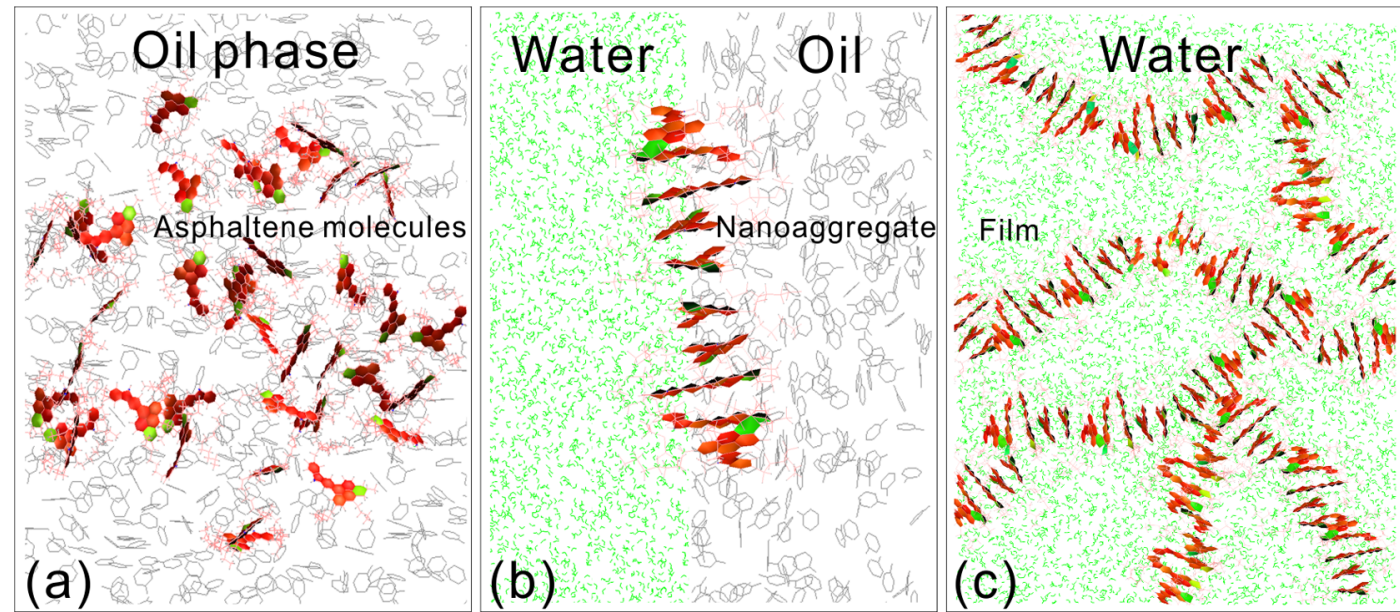

Figure 7. Schematic view of the formation of the asphaltene protecting film at the oil/water interface. (a) Initially, the asphaltene molecules are randomly distributed in solvent. (b) A few asphaltene molecules assemble to form nanoaggregates with the molecular PAH planes face-to-face stacked. (c) Fence-like asphaltene aggregates perpendicularly pinned on the water surface and knitting together to form the protecting film.

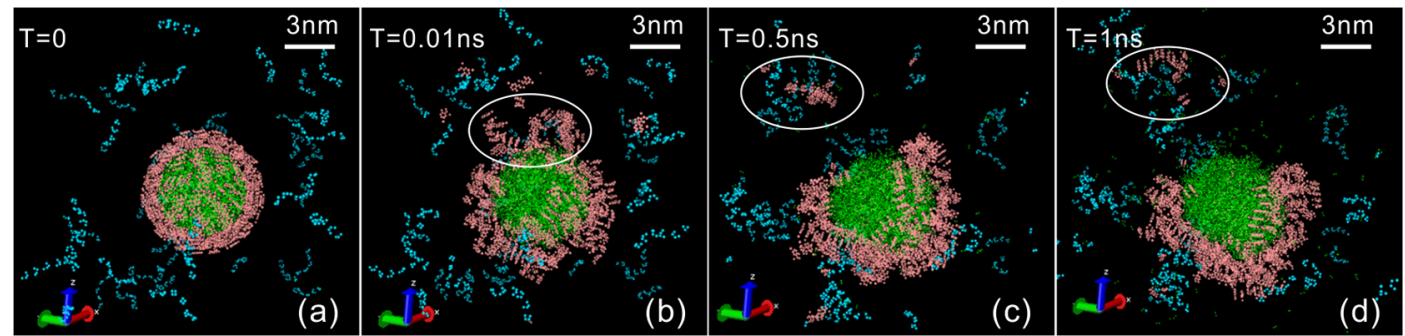

Figure 8. Sample snapshots of the demulsification processes by EC. The EC molecules encroach on the asphaltenes film and gradually pull off the fence-like structure, like peeling an orange. Water, EC, and coal asphaltene molecules are represented in green, light blue, and pink, respectively. The toluene molecules are hidden.

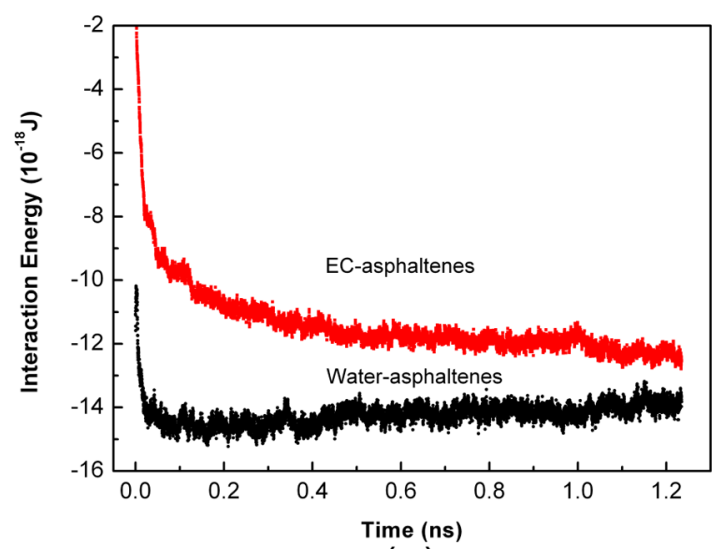

(a)
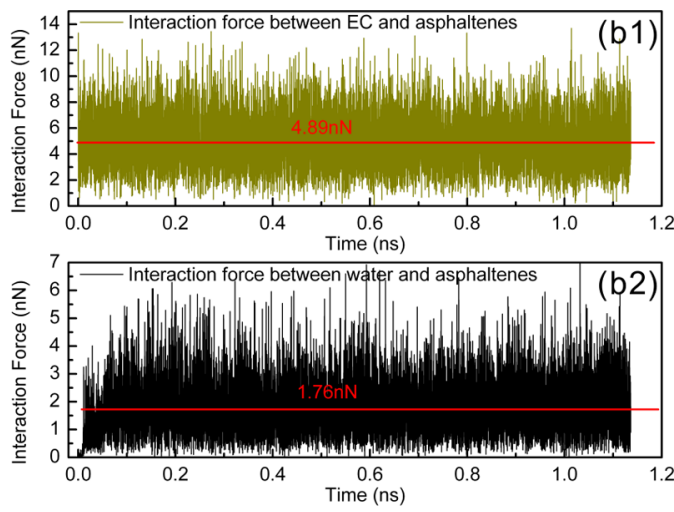

(b)

Figure 9. (a) Interaction energy and (b) force for water/asphaltenes and EC/asphaltenes at a toluene/water interface.

The asphaltene nanoaggregate generally consists of a few molecules, with the molecular PAHs face-to-face stacking together. Moreover, most of the $\mathrm{PAH}$ planes were found perpendicular to the water surface (Figures 4 and 5). If the asphaltene molecules are considered as "stakes" (Figure 7a), then the asphaltene nanoaggregate could be regarded as a "fence" (Figure 7b), which is perpendicularly pinned on the water surface. Probably with the help of the peripheral alkyl group or even other compounds such as resins, multiple fencelike nanoaggregates twist and knit together at the oil/water interface to form a steady thin film (Figure 7c), which prevents the isolated water droplets suspended in oil from coalescencing (Figure S7). The good stability of the water/oil emulsions is thereby well understood.

3.4. Demulsification of the Crude Oil Emulsion. Ethyl cellulose has been reported to be an effective demulsifier for water-in-diluted bitumen emulsions. ${ }^{17,63}$ In this work, EC with a lower polymerization (the degrees of substitution of ethoxylation and polymerization are 2.7 and 6, respectively) was used as the demulsifier for simulation of the process of demulsification of a water/asphaltene/toluene emulsion system. As shown in Figure 8a, 50 EC molecules (123.6 g/L) were 
dissolved in toluene. The EC molecules then gradually approached the asphaltenes at the toluene/water interface (Figure $8 \mathrm{~b}$ ). It was interesting to find that, subsequently, the EC molecules encroached on the asphaltenes film and gradually pulled out the fence-like structure like peeling an orange (Figure 8c). As a result, the water droplet was partially uncovered, and so the coalescence of water droplets could occurred.

To understand the effect of EC on the asphaltene protective film, the RDF of asphaltenes with EC at toluene/water interface was calculated and is shown in Figure 6a (the red plot). Compared to the situation without EC (the black plot), the demulsifier played a significant role with the asphaltenes aggregates. It is observed that the strength of the peaks at about 7.7 and $11 \AA$ increased considerably, indicating that there is an increase in the distance between the PAHs owing to the external pulling force from EC. The asphaltene/EC and asphaltene/water interactions were calculated and are shown in Figure 9. It is shown that the interaction energy between asphaltenes and water became nearly constant after $0.5 \mathrm{~ns}$ (Figure 9a). For asphaltenes and EC, the interaction energy decreased, owing to the approach of EC to the water surface. When EC diffused to the water surface and interacted with the asphaltenes, the interaction energy tended to be stable after 0.8 $\mathrm{ns}$, and the system reached its equilibrium. The interaction forces for asphaltenes/EC and asphaltenes/water are shown in Figure $9 \mathrm{~b}$. The average asphaltene/EC interaction force is about $4.89 \mathrm{nN}$, which is larger than that between asphaltene and water, $1.76 \mathrm{nN}$. Obviously, the $\mathrm{EC}$ molecules predominated over the water molecules to drag away the asphaltenes from the water surface. Therefore, the asphaltene protective film was partially destroyed, resulting in exposure of the water droplet.

The slope of mean-squared displacement (MSD) versus time is proportional to the diffusion coefficient of molecules. The diffusion coefficients of molecules in the simulation systems could be calculated from the simulation trajectories by using the Einstein equation:

$$
D=\frac{1}{2 N_{\mathrm{d}}} \lim _{t \rightarrow \infty} \frac{\mathrm{d}}{\mathrm{d} t}\left\langle\overrightarrow{\mid r(t)}-\left.\overrightarrow{r(0)}\right|^{2}\right\rangle
$$

where $N_{\mathrm{d}}$ is the dimensionality $\left(N_{\mathrm{d}}=3\right.$ for the simulations), and $\overrightarrow{r(t)}$ and $\left\langle\overrightarrow{r(t)}-\left.\overrightarrow{r(0)}\right|^{2}\right\rangle$ are the position and squared displacement of given molecules at time $t$, respectively. The results in Figure 10 show that the diffusion coefficient of a coal asphaltene nanoaggregate at a toluene/water interface is about $1.19 \times 10^{-10} \mathrm{~m}^{2} / \mathrm{s}$, which is in agreement with the previous calculation for asphaltene at a toluene/water interface. ${ }^{34}$ In addition, the MSD plot of coal asphaltene with EC was initially analogous to that of asphaltene without EC (before $0.2 \mathrm{~ns}$ ). The MSD of asphaltene with EC then gradually became larger (about $0.2-0.7 \mathrm{~ns}$ ). At last, it was nearly linear with respect to time after $0.7 \mathrm{~ns}$. At that time, the diffusion coefficient of asphaltene nanoaggregates with EC at the toluene/water interface was $2.17 \times 10^{-9} \mathrm{~m}^{2} / \mathrm{s}$. Clearly, the diffusion velocity of the asphaltene nanoaggregates at the toluene/water interface was accelerated by attaching the EC molecules.

\section{CONCLUSION}

MD simulations were employed to explore the aggregation and orientation behaviors of the asphaltene molecules in vacuum or at various water surfaces. It was found that the initially

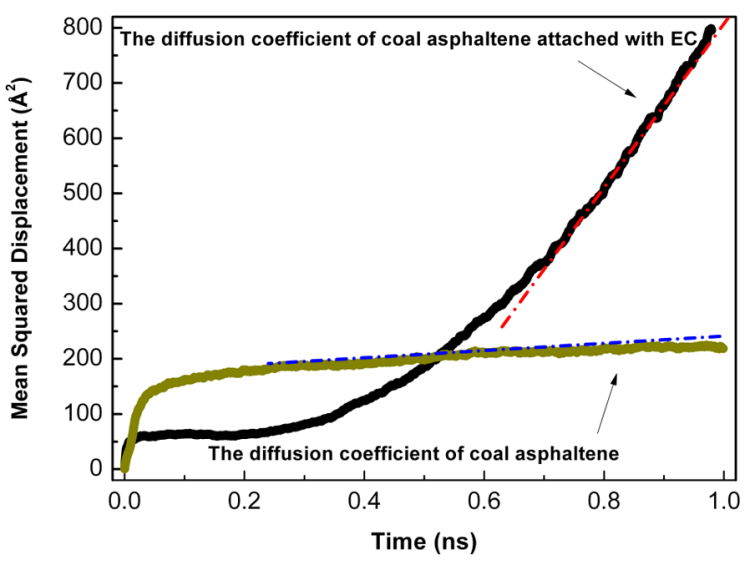

Figure 10. Mean-squared displacement of the coal asphaltenes and coal asphaltenes attached with EC at the toluene/water interface.

disordered asphaltene molecules at vacuum/toluene quickly self-assembled into ordered aggregates consisting of several molecules, in which the asphaltene aromatic rings face-to-face stacked together. Such aggregation behavior could occur at the vacuum/water or toluene/water interface as well. More importantly, it was interesting to find that the stacked $\mathrm{PAH}$ planes prefer to be perpendicular to the water surface. This finding was supported by the statistical analysis of the asphaltenes' orientation on the water surface. The formation process of the asphaltene protective film from the randomly distributed asphaltene molecules was demonstrated in the MD simulation. In addition, the demulsification process, using a chemical demulsifier, was studied. It was observed that the asphaltene protective film was destroyed by the demulsifier of EC molecules, leading to exposure of the water droplet. These findings indicate that $\mathrm{MD}$ simulation is a useful and effective way to study the mechanism of stabilization and demulsification of the water/oil emulsion at a molecular scale. The results obtained in this work will be of significance in guiding the development of demulsification technology.

\section{ASSOCIATED CONTENT}

\section{S Supporting Information}

Details of additional simulations to validate the molecular model and force field, energies of the simulation system to indicate the equilibrium states in a given time step, and dynamic pictures of the simulation. This material is available free of charge via the Internet at http://pubs.acs.org.

\section{AUTHOR INFORMATION}

\section{Corresponding Author}

*Tel.: 86-931-4968051. Fax: 86-931-8277088. E-mail: slren@ licp.cas.cn.

\section{Notes}

The authors declare no competing financial interest.

\section{ACKNOWLEDGMENTS}

The authors acknowledge financial support from the National Natural Science Foundation of China (Grant 51374195) and the "100 Talents Program" held by the Chinese Academy of Sciences (CAS). 


\section{REFERENCES}

(1) Spiecker, P. M.; Kilpatrick, P. K. Interfacial rheology of petroleum asphaltenes at the oil-water interface. Langmuir 2004, 20 (10), 40224032.

(2) Speight, J. In Catalysis on the energy scene; Kaliaguine, S., Mahaffy, A., Eds.; Elsevier, Amsterdam, 1984.

(3) McLean, J. D.; Kilpatrick, P. K. Effects of asphaltene aggregation in model heptane-toluene mixtures on stability of water-in-oil emulsions. J. Colloid Interface Sci. 1997, 196 (1), 23-34.

(4) McLean, J. D.; Kilpatrick, P. K. Effects of asphaltene solvency on stability of water-in-crude-oil emulsions. J. Colloid Interface Sci. 1997, 189 (1), 242-253.

(5) McLean, J. D.; Spiecker, P. M.; Sullivan, A. P.; Kilpatrick, P. K. The role of petroleum asphaltenes in the stabilization of water-in-oil emulsions. In Structure and Dynamics of Asphaltenes; Mullins, O. C., Sheu, E. Y., Eds.; Springer: Berlin, 1998; pp 377-422.

(6) Angle, C. W.; Hamza, H. A. Drop sizes during turbulent mixing of toluene-heavy oil fractions in water. AIChE J. 2006, 52 (7), 26392650.

(7) Angle, C. W.; Hamza, H. A.; Dabros, T. Size distributions and stability of toluene diluted heavy oil emulsions. AIChE J. 2006, 52 (3), 1257-1266.

(8) Buckley, J. S.; Liu, Y.; Xie, X.; Morrow, N. R. Asphaltenes and crude oil wetting - the effect of oil composition. SPE J. 1997, 2 (2), 107-119.

(9) Ali Mansoori, G. Modeling of asphaltene and other heavy organic depositions. J. Petrol. Sci. Eng. 1997, 17 (1), 101-111.

(10) Umutbaev, V.; Efimova, A.; Fryazinov, V.; Sapozhnikova, E.; Savkova, V. Protection of equipment from corrosion. Chem. Technol. Fuels Oils 1981, 17 (6), 330-337.

(11) Silverman, L.; Winkler, S.; Tiethof, J.; Witoshkin, A. Matrix effects in catalytic cracking; National Petroleum Refiners Association, Washington, DC, 1986.

(12) Rahmani, N. H. G.; Dabros, T.; Masliyah, J. H. Online optical monitoring of asphaltene aggregation. Ind. Eng. Chem. Res. 2005, 44 (1), 75-84.

(13) Yeung, A.; Dabros, T.; Masliyah, J. Does equilibrium interfacial tension depend on method of measurement? J. Colloid Interface Sci. 1998, 208 (1), 241-247.

(14) Moran, K.; Yeung, A.; Masliyah, J. Measuring interfacial tensions of micrometer-sized droplets: a novel micromechanical technique. Langmuir 1999, 15 (24), 8497-8504.

(15) Yeung, A.; Dabros, T.; Masliyah, J.; Czarnecki, J. Micropipette: a new technique in emulsion research. Colloids Surf. A-Physicochemistry 2000, 174 (1-2), 169-181.

(16) Moran, K.; Czarnecki, J. Competitive adsorption of sodium naphthenates and naturally occurring species at water-in-crude oil emulsion droplet surfaces. Colloids Surf. A-Physicochemistry 2007, 292 (2), 87-98.

(17) Feng, X.; Xu, Z.; Masliyah, J. Biodegradable polymer for demulsification of water-in-bitumen emulsions. Energy Fuels 2008, 23 (1), 451-456.

(18) Feng, X.; Mussone, P.; Gao, S.; Wang, S.; Wu, S. Y.; Masliyah, J. $\mathrm{H}$.; $\mathrm{Xu}, \mathrm{Z}$. Mechanistic study on demulsification of water-in-diluted bitumen emulsions by ethylcellulose. Langmuir 2009, 26 (5), 30503057.

(19) Ese, M. H.; Sjöblom, J.; Djuve, J.; Pugh, R. An atomic force microscopy study of asphaltenes on mica surfaces. Influence of added resins and demulsifiers. Colloid Polym. Sci. 2000, 278 (6), 532-538.

(20) Zhang, L. Y.; Xu, Z.; Masliyah, J. H. Characterization of adsorbed Athabasca asphaltene films at solvent-water interfaces using a Langmuir interfacial trough. Ind. Eng. Chem. Res. 2005, 44 (5), 11601174.

(21) Wang, S.; Liu, J.; Zhang, L.; Xu, Z.; Masliyah, J. Colloidal interactions between asphaltene surfaces in toluene. Energy Fuels 2008, 23 (2), 862-869.

(22) Orbulescu, J.; Mullins, O. C.; Leblanc, R. M. Surface chemistry and spectroscopy of UG8 asphaltene Langmuir film, part 1. Langmuir 2010, 26 (19), 15257-15264.
(23) Orbulescu, J.; Mullins, O. C.; Leblanc, R. M. Surface chemistry and spectroscopy of UG8 asphaltene Langmuir film, Part 2. Langmuir 2010, 26 (19), 15265-15271.

(24) Merdrignac, I.; Espinat, D. Physicochemical characterization of petroleum fractions: the state of the art. Oil Gas Sci. Technol. 2007, 62 (1), 7-32.

(25) Rogel, E. Studies on asphaltene aggregation via computational chemistry. Colloids Surf. A-Physicochemistry 1995, 104 (1), 85-93.

(26) Rogel, E. Simulation of interactions in asphaltene aggregates. Energy Fuels 2000, 14 (3), 566-574.

(27) Pacheco-Sánchez, J.; Zaragoza, I.; Martinez-Magadan, J. Asphaltene aggregation under vacuum at different temperatures by molecular dynamics. Energy Fuels 2003, 17 (5), 1346-1355.

(28) Pacheco-Sánchez, J.; Alvarez-Ramirez, F.; Martinez-Magadan, J. Morphology of aggregated asphaltene structural models. Energy Fuels 2004, 18 (6), 1676-1686.

(29) Takanohashi, T.; Sato, S.; Saito, I.; Tanaka, R. Molecular dynamics simulation of the heat-induced relaxation of asphaltene aggregates. Energy Fuels 2003, 17 (1), 135-139.

(30) Sedghi, M.; Goual, L.; Welch, W.; Kubelka, J. Effect of asphaltene structure on association and aggregation using molecular dynamics. J. Phys. Chem. B 2013, 117 (18), 5765-5776.

(31) Ungerer, P.; Rigby, D.; Leblanc, B.; Yiannourakou, M. Sensitivity of the aggregation behaviour of asphaltenes to molecular weight and structure using molecular dynamics. Mol. Simul. 2014, 40 $(1-3), 115-122$.

(32) Zhang, L.; Greenfield, M. L. Molecular orientation in model asphalts using molecular simulation. Energy Fuels 2007, 21 (2), 11021111.

(33) Kuznicki, T.; Masliyah, J. H.; Bhattacharjee, S. Molecular dynamics study of model molecules resembling asphaltene-like structures in aqueous organic solvent systems. Energy Fuels 2008, 22 (4), 2379-2389.

(34) Kuznicki, T.; Masliyah, J. H.; Bhattacharjee, S. Aggregation and partitioning of model asphaltenes at toluene-water interfaces: molecular dynamics simulations. Energy Fuels 2009, 23 (10), 50275035.

(35) Teklebrhan, R. B.; Ge, L.; Bhattacharjee, S.; Xu, Z.; Sjöblom, J. Initial partition and aggregation of uncharged polyaromatic molecules at the oil-water interface: a molecular dynamics simulation study. J. Phys. Chem. B 2014, 118, 1040-1051.

(36) Mikami, Y.; Liang, Y.; Matsuoka, T.; Boek, E. S. Molecular dynamics simulations of asphaltenes at the oil-water interface: from nanoaggregation to thin-film formation. Energy Fuels 2013, 27 (4), $1838-1845$.

(37) Alvarez, F.; Flores, E.; Castro, L.; Hernández, J.; López, A.; Vázquez, F. Dissipative particle dynamics (DPD) study of crude oilwater emulsions in the presence of a functionalized co-polymer. Energy Fuels 2011, 25, 562-567.

(38) Martínez, L.; Andrade, R.; Birgin, E.; Martínez, J. Packmol: A package for building initial configurations for molecular dynamics simulations. J. Comput. Chem. 2009, 30 (13), 2157-2164.

(39) Boek, E. S.; Yakovlev, D. S.; Headen, T. F. Quantitative molecular representation of asphaltenes and molecular dynamics simulation of their Aggregation. Energy Fuels 2009, 23 (3), 12091219.

(40) Sheremata, J. M.; Gray, M. R.; Dettman, H. D.; McCaffrey, W. C. Quantitative molecular representation and sequential optimization of Athabasca asphaltenes. Energy Fuels 2004, 18 (5), 1377-1384.

(41) Mullins, O. C.; Hammami, A.; Marshall, A. G. Asphaltenes, heavy oils, and petroleomics; Springer: New York, 2007; p 49.

(42) Groenzin, H.; Mullins, O. C. Molecular size and structure of asphaltenes from various sources. Energy Fuels 2000, 14 (3), 677-684.

(43) Berendsen, H. J. C.; Grigera, J. R.; Straatsma, T. P. The missing term in effective pair potentials. J. Phys. Chem. 1987, 91 (24), 62696271.

(44) Dauber-Osguthorpe, P.; Roberts, V. A.; Osguthorpe, D. J.; Wolff, J.; Genest, M.; Hagler, A. T. Structure and energetics of ligand binding to proteins: Escherichia coli dihydrofolate reductase- 
trimethoprim, a drug-receptor system. Proteins: Struct., Funct. Bioinf.

1988, 4 (1), 31-47.

(45) Plimpton, S. Fast parallel algorithms for short-range molecular dynamics. J. Comput. Phys. 1995, 117 (1), 1-19.

(46) Hoover, W. G. Canonical dynamics: equilibrium phase-space distributions. Phys. Rev. A 1985, 31 (3), 1695-1697.

(47) Humphrey, W.; Dalke, A.; Schulten, K. VMD: Visual molecular dynamics. J. Mol. Graphics 1996, 14 (1), 33-38.

(48) Kell, G. S. Density, thermal expansivity, and compressibility of liquid water from 0 deg to $150 \mathrm{deg}$. Correlations and tables for atmospheric pressure and saturation reviewed and expressed on 1968 temperature scale. J. Chem. Eng. Data 1975, 20 (1), 97-105.

(49) Kashiwagi, H.; Hashimoto, T.; Tanaka, Y.; Kubota, H.; Makita, T. Thermal conductivity and density of toluene in the temperature range $273-373 \mathrm{~K}$ at pressures up to $250 \mathrm{MPa}$. Int. J. Thermophys. 1982, 3 (3), 201-215.

(50) Allen, M. P.; Tildesley, D. J. Computer simulation of liquids; Oxford University Press: Oxford, UK, 1989.

(51) Wang, J. H. Self-diffusion coefficients of water. J. Phys. Chem. 1965, 69 (12), 4412-4412.

(52) Yuan, S.; Chen, Y.; Xu, G. Molecular dynamics studies on octadecylammonium chloride at the air/liquid interface. Colloids Surf. A-Physicochemistry 2006, 280 (1-3), 108-115.

(53) Sztukowski, D. M.; Yarranton, H. W. Rheology of asphaltenetoluene/water interfaces. Langmuir 2005, 21 (25), 11651-11658.

(54) Mullins, O. C. The modified Yen model. Energy Fuels 2010, 24 (4), 2179-2207.

(55) Andrews, A. B.; McClelland, A.; Korkeila, O.; Demidov, A.; Krummel, A.; Mullins, O. C.; Chen, Z. Molecular orientation of asphaltenes and $\mathrm{PAH}$ model compounds in Langmuir-Blodgett films using sum frequency generation spectroscopy. Langmuir 2011, 27 (10), 6049-6058.

(56) Rane, J. P.; Harbottle, D.; Pauchard, V.; Couzis, A.; Banerjee, S. Adsorption kinetics of asphaltenes at the oil-water interface and nanoaggregation in the bulk. Langmuir 2012, 28 (26), 9986-9995.

(57) Sheu, E. Y.; De Tar, M. M.; Storm, D. A.; DeCanio, S. J. Aggregation and kinetics of asphaltenes in organic solvents. Fuel 1992, 71 (3), 299-302.

(58) Andersen, S. I.; Christensen, S. D. The critical micelle concentration of asphaltenes as measured by calorimetry. Energy Fuels 2000, 14 (1), 38-42.

(59) Zhang, L. Y.; Lopetinsky, R.; Xu, Z.; Masliyah, J. H. Asphaltene monolayers at a toluene/water interface. Energy Fuels 2005, 19 (4), $1330-1336$

(60) Solovyev, A.; Zhang, L. Y.; Xu, Z.; Masliyah, J. H. Langmuir films of bitumen at oil/water interfaces. Energy Fuels 2006, 20 (4), $1572-1578$.

(61) Yeung, A.; Dabros, T.; Czarnecki, J.; Masliyah, J. On the interfacial properties of micrometre-sized water droplets in crude oil. Proc. R. Soc. London, Ser. A: Math., Phys. Eng. Sci. 1999, 455 (1990), 3709-3723.

(62) Dabros, T.; Yeung, A.; Masliyah, J.; Czarnecki, J. Emulsification through area contraction. J. Colloid Interface Sci. 1999, 210 (1), 222224.

(63) Feng, X.; Wang, S.; Hou, J.; Wang, L.; Cepuch, C.; Masliyah, J.; $\mathrm{Xu}, \mathrm{Z}$. Effect of hydroxyl content and molecular weight of biodegradable ethylcellulose on demulsification of water-in-diluted bitumen emulsions. Ind. Eng. Chem. Res. 2011, 50 (10), 6347-6354. 